Supporting Information

\title{
Highly Selective Electrochemical Detection of 5- Formyluracil Relying on (2-Benzimidazolyl) Acetonitrile Labeling
}

Jing Tang ${ }^{\mathrm{a}, \mathrm{c}, 1}$, Guangrong Zou ${ }^{\mathrm{b}, 1}$, Chen Chen ${ }^{\mathrm{a}}$, Jing Ren ${ }^{\mathrm{a}}$, Fang Wang ${ }^{\mathrm{a}, \mathrm{c}} *$, Zilin Chen ${ }^{\mathrm{a}, \mathrm{c}} *$

${ }^{a}$ Key Laboratory of Combinatorial Biosynthesis and Drug Discovery, Ministry of Education, Hubei Province Engineering and Technology Research Center for Fluorinated Pharmaceuticals, and Wuhan University School of Pharmaceutical Sciences, Wuhan, 430071, China

${ }^{\mathrm{b}}$ Key Laboratory of Biomedical Polymers of Ministry of Education, the Institute for Advanced Studies, Hubei Province Key Laboratory of Allergy and Immunology, Wuhan University School of Chemistry and Molecular Sciences, Wuhan, 430072, China

${ }^{c}$ State Key Laboratory of Transducer Technology, Chinese Academy of Sciences, Beijing, 100080, China

*:Corresponding authors.

Ass. Prof. Fang Wang Tel: 86-27-68759829, fax: 86-27-68759850.

E-mail address: fwang@whu.edu.cn

Prof. Zilin Chen.

Tel: 86-27-68759893, fax: 86-27-68759850.

E-mail address: chenzl@whu.edu.cn 


\section{Table of Contents}

1. Models of oligonucleotides sequences $\quad$ S3

2. Performance comparison of different methods $\quad$ S3

3. Recovery determination of ODN-5fU in mixed samples

4. Detection of $5 \mathrm{fU}$ in $\gamma$-irradiated DNA $\quad$ S4

5. XPS of Au-S bond

6. Reaction formula between azi-biaU and DBCO-PEG4-Biotin S5

7. MALDI-TOF-MS analysis of ODN-Biotin-U S6

8. MALDI-TOF-MS analysis of ODN-Biotin-U after reaction with T4 PNK S6

$\begin{array}{ll}\text { 9. Nyquist plots of the modified electrodes } & \text { S7 }\end{array}$

$\begin{array}{ll}\text { 10.Stability } & \text { S7 }\end{array}$

$\begin{array}{ll}\text { 11.References } & \text { S8 }\end{array}$ 
Table S1. Models of oligonucleotides sequences.

\begin{tabular}{cc}
\hline Oligomer & Sequence (from 5' to $3^{\prime}$ ) \\
ODN-T & GACTCAATAGCCGTA \\
ODN-5fU & GACTCAA5fUAGCCGTA \\
ODN-5fC & GACTCAA5fCAGCCGTA \\
ODN-AP & GACTCAAAPAGCCGTA \\
ODN-T & TCGACTGTGTGTATCTGTGTGAGTCGAG \\
ODN-5fU & TCGACTGTGTGTATC5fUGTGTGAGTCGAG \\
ODN-5fC & TCGACTGTGTGTATC5fCGTGTGAGTCGAG \\
ODN-AP & CCGACTGTGGTATCAPGTGTGAGTCGAG \\
Complementary Strand &
\end{tabular}

Table S2. Analytical performance of different methods for pyrimidine modifications.

\begin{tabular}{cccccc}
\hline Method & Analyte & Selectivity & Linear Range & LOD & Refs \\
\hline Fluorescent labeling & $5 \mathrm{fU}$ & yes & $20-1000 \mathrm{nM}$ & - & {$[31]$} \\
Fluorescent labeling & Aldehydes & no & $10-50 \mathrm{pM}$ & - & {$[32]$} \\
Fluorescent labeling & $5 \mathrm{fU}$ & yes & $1-100 \mathrm{pmol}$ & - & {$[33]$} \\
Fluorescent labeling & $5 \mathrm{fC}$ & - & $1-100 \mathrm{nM}$ & - & {$[45]$} \\
LC-MS/MS & $5 \mathrm{fU}$ & no & - & $3-4 \mathrm{fmol}$ & {$[30]$} \\
LC-ESI/MS/MS & $5 \mathrm{hmC}$ & no & - & $0.04 \mathrm{fmol}$ & {$[46]$} \\
Electrochemical detection & $5 \mathrm{fU}$ & yes & $0.1-10 \mathrm{nM}$ & $0.075 \mathrm{nM}$ & this work \\
\hline
\end{tabular}

"-" means the information is not indicated in the article. 
Table S3. Recovery determination of ODN-5fU in mixed samples.

\begin{tabular}{cccc}
\hline Added (nM) & Determination $(\mathrm{nM})$ & Recovery $(\%)$ & RSD $(\%, \mathrm{~N}=3)$ \\
\hline 2.50 & 2.34 & 93.60 & 3.47 \\
1.50 & 1.66 & 110.60 & 4.83 \\
0.50 & 0.46 & 92.00 & 6.94 \\
\hline
\end{tabular}

Table S4. The number of 5fU in $\gamma$-irradiated DNA obtained by different methods.

\begin{tabular}{ccc}
\hline Method & $5 \mathrm{fU} \cdot 10^{-6}$ bases $\cdot \mathrm{Gy}^{-1}$ & Refs \\
\hline Fluorescent labeling & 8.9 & {$[33]$} \\
LC-MS & 15.3 & {$[44]$} \\
LC-MS & 6.2 & this work \\
Electrochemical detection & 5.3 & this work \\
\hline
\end{tabular}




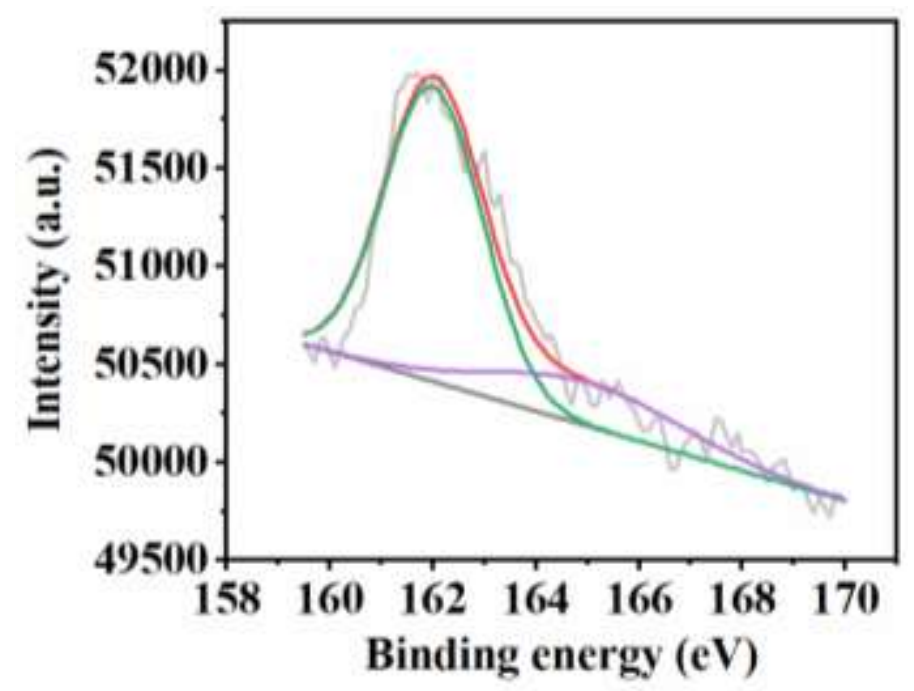

Figure S1. XPS analysis of sulfur element in Au-S bond (green curve) and in sulfhydryl group (grape curve) on the surface of AuNPs/GCE immobilized with sulfhydryl modified ODN.

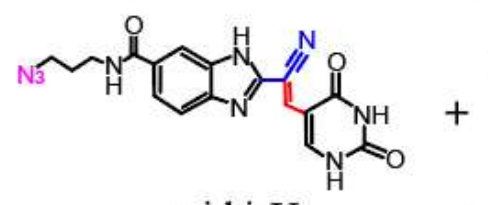

azi-biaU

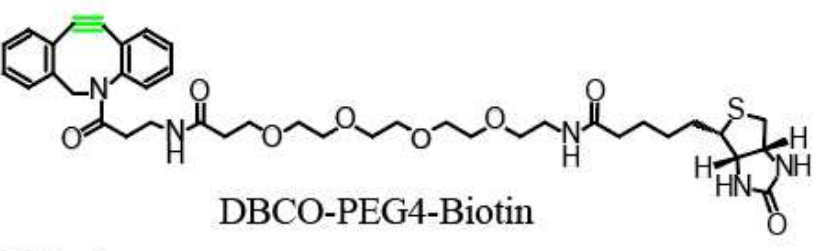

$37^{\circ} \mathrm{C}, 2 \mathrm{~h}$

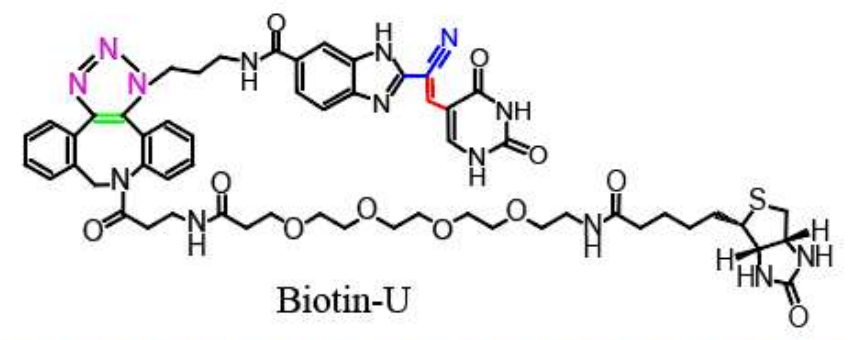

Figure S2. The reaction formula between azi-biaU and DBCO-PEG4-Biotin. 


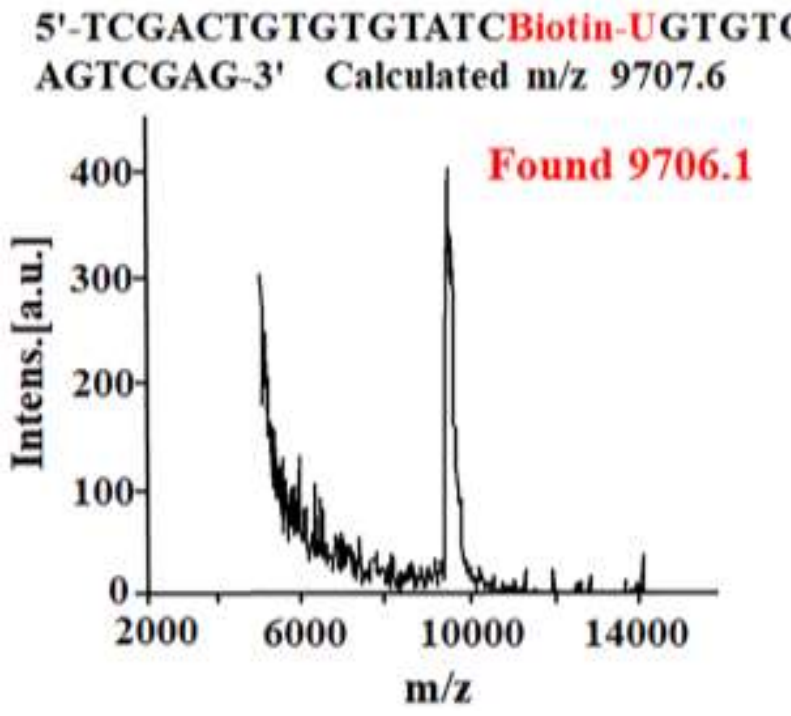

Figure S3. MALDI-TOF-MS analysis of ODN-azi-biaU after further reaction with DBCO-PEG4-Biotin ( (denoted as ODN-Biotin-U).

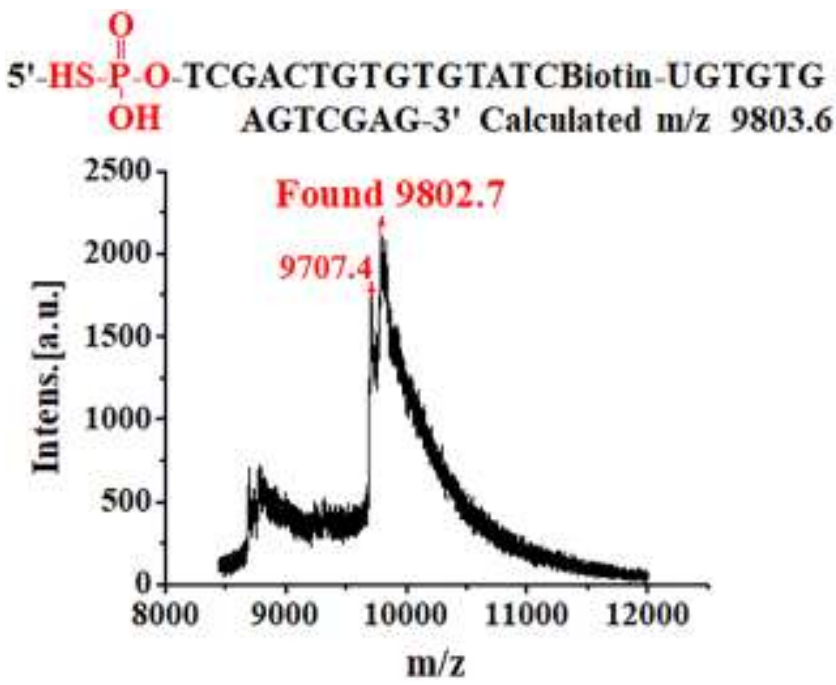

Figure S4. MALDI-TOF-MS analysis of ODN-Biotin-U after further reaction with T4 PNK. 


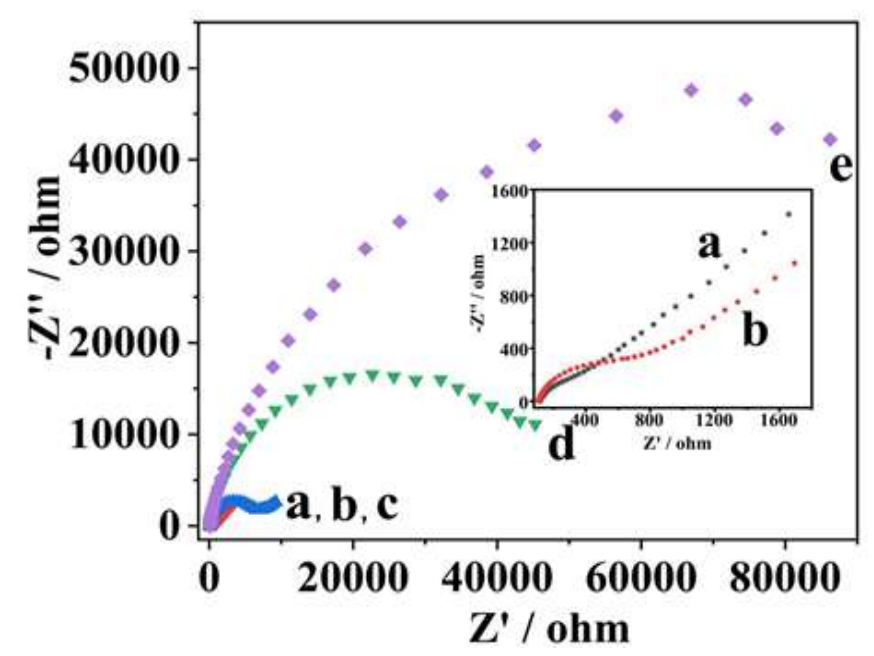

Figure S5. Nyquist plots of (a) AuNPs/GCE, (b) ODN-5fU/AuNPs/GCE, (c) MCH/ODN-5fU/AuNPs/GCE，(d) BSA/MCH/ODN-5fU/AuNPs/GCE， (e) SA$\mathrm{HRP} / \mathrm{BSA} / \mathrm{MCH} / \mathrm{ODN}-5 \mathrm{fU} / \mathrm{AuNPs} / \mathrm{GCE}$ in $0.1 \mathrm{M} \mathrm{KCl}$ solution containing $1 \mathrm{mM}$ $\left[\mathrm{Fe}(\mathrm{CN})_{6}\right]^{3-1 / 4}$.

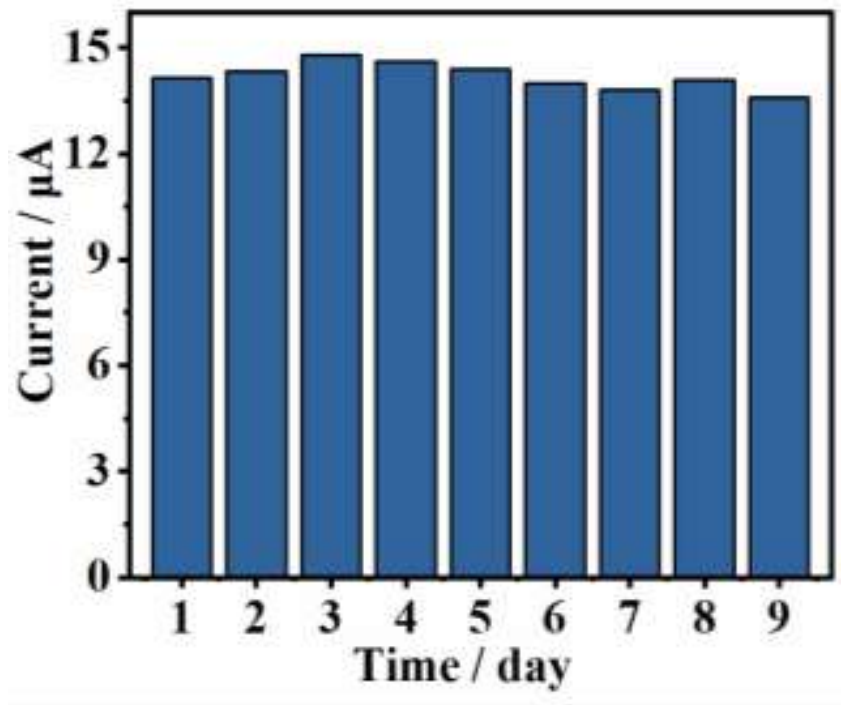

Figure S6. Changes in electrochemical signals over time, double strand, $0.01 \mu \mathrm{M}$. 


\section{References}

(30)Hong, H.; Wang, Y. Derivatization with Girard reagent T combined with LCMS/MS for the sensitive detection of 5-formyl-2'-deoxyuridine in cellular DNA. Anal. Chem. 2007, 79, 322-326. https://doi.org/10.1021/ac061465w

(31)Liu, C.; Chen, Y.; Wang, Y.; Wu, F.; Zhang, X.; Yang, W.; Wang, J.; Chen, Y.; He, Z.; Zou, G.; Wang, S.; Zhou, X. A highly efficient fluorescence-based switch-on detection method of 5-formyluracil in DNA. Nano Res. 2017, 10, 2449-2458. https://doi.org/10.1007/s12274-017-1445-2

(32)Liu, C.; Luo, X.; Chen, Y.; Wu, F.; Yang, W.; Wang, Y.; Zhang, X.; Zou, G.; Zhou, X. Selective Labeling Aldehydes in DNA.Anal. Chem. 2018, 90, 14616-14621. https://doi.org/10.1021/acs.analchem.8b04822

(33)Hirose, W.; Sato, K.; Matsuda, A. Selective Detection of 5-Formyl-2'-deoxyuridine, an Oxidative Lesion of Thymidine, in DNA by a Fluorogenic Reagent. Angew. Chem.Int. Edit. 2010, 49, 8392-8394. https://doi.org/10.1002/anie.201004087

(44)Frelon, S.; Douki, T.; Ravanat, J. L.; Pouget, J. P.; Tornabene, C.; Cadet, J. Highperformance liquid chromatography-tandem mass spectrometry measurement of radiation-induced base damage to isolated and cellular DNA. Chem. Res. Toxicol. 2000, 13, 1002-1010. https://doi.org/10.1021/tx000085h

(45)Hu, J.; Xing, X.; Xu, X.; Wu, F.; Guo, P.; Yan, S.; Xu, Z.; Xu, J.; Weng, X.; Zhou, X. Selective chemical labelling of 5-formylcytosine in DNA by fluorescent dyes. Chem.-Eur. J. 2013, 19, 5836-5840. https://doi.org/10.1002/chem.201300082 (46)Zhang, H.; Xiong, J.; Qi, B.; Feng, Y.; Yuan, B. The existence of 5hydroxymethylcytosine and 5-formylcytosine in both DNA and RNA in mammals. Chem. Commun. 2016, 52, 737-740. https://doi.org/10.1039/c5cc07354e 